\title{
Research on Joint of Simulation and Sea Test for Sonar Operational Range
}

\author{
Guoqiang Ma
}

Sci. \& Tech. on Underwater Acoustic Antaganizing Laboratory, Zhanjiang 524022, P. R. China

mgq421@foxmail.com

Keywords: sonar system, sonar operational Range, joint test.

\begin{abstract}
Sonar operational range is an important tactics and technique parameter for sonar system. It is a variable number because of many factors affecting in the actual using, so it brings more complex in the test. Being analyzing the influencing factors for sonar operational range, this paper summarizes the traditional test. Joint of simulation and sea test for sonar operational range is discussed on the base of analyzing the localization of traditional method and the demand of sonar developing and using.
\end{abstract}

\section{Factors analyzing of affecting sonar operational range}

Sonar operational range is an important tactics and technique parameter for sonar system. As the sonar's working environment is a complicated underwater acoustic environment, the actual sonar operational range is a synthetical index which depends on the performance of sonar system, environment of the underwater acoustic propagation and target characteristics.

Sonar equation is a useful tool in designing the sonar system. It is usually used to analyze and attest the performance of sonar system. It forms an organic structure of hydrophone, underwater sound channel, noise, reverberation, detection and signal designing. Active and passive sonar equation is as follows,

Active sonar equation (Reverberation Limited),

$S L-2 T L+T S=R L+D$

Active sonar equation (Noise Limited),

$S L-2 T L+T S=N L-(G S+G T)+D T$

Passive sonar equation,

$S L-T L=N L-(G S+G T)+D T$

In the above sonar equations, there are four kind parameters ${ }^{[1]}$,

a) Parameters dependent on the sonar system itself

These parameters are confirmed by designing the sonar system. They determine the inherent performance of sonar system. They are including emission Sound Level SL ,Gain Spatial GS, Gain Time GT and Detection Threshold $D T$.

b) Parameters dependent on the detected target

These parameters are including target radiated Noise Level, Target Strength TS .

c) Parameters dependent on the underwater acoustic environment

These parameters are including Transmission Loss $T L$, ambient Noise Level $N L$.

d) Parameter which dependent on the sonar system and sonar's working environment

This parameter is mainly including Reverberation Level $R L$.

Sonar operational range is a proper tactics index. It is not designed by engineers. It embodiments the ability of sonar system working in different environment (including different target, different platform, different underwater acoustic propagation environment). Without thinking of the 
underwater acoustic environment, the Factor Of Merit (FOM ) to the sonar system embodiment the ability of sonar system itself. The FOM of active and passive sonar is as follows,

$$
\text { FOM of active sonar: } F O M=(S L+T S-N L+G S+G T-D T) / 2
$$

FOM of passive sonar: $F O M=S L-N L+G S+G T-D T$

We can determine the farthest sonar operational range by the equation $F O M=T L$. Obviously, for the specifical target, more larger the FOM , more farther the sonar operational range. As the same FOM , the sonar operational range is not the same for different underwater acoustic propagation environment. The sonar operational range is far for good underwater acoustic propagation environment and is near for bad underwater acoustic propagation environment. The goal of sonar designer is to design a sonar system which is the largest FOM for the specifical target characteristics and sonar platform.

In actual operational using, with the variety of target characteristics, sonar platform and underwater acoustic propagation channel, the sonar operational range will be different. For a fixed parameters sonar system, the factors which influence the sonar operational range are including target characteristics, sonar platform and underwater acoustic propagation channel.

Although the FOM of sonar system embodiment the ability of sonar system itself, it is not a tactics index. Because the sonar operational range is a variable number, the critical department should test the sonar operational range in the condition of one state.

\section{Tradition test method for sonar operational range}

For a finished sonar system, the critical department tests the sonar operational range in the condition of sea test. In the actual sea test, there is three times chance to test.If the condition of sea test is not satisfaction with the limited condition of sonar operational range. It is permitted to convert the condition of sea test to limited condition of sonar operational range.

Test rule for sonar operational range. The index of sonar operational range is from sonar equation. We estimate the sonar operational range based on signal to noise ratio exceeding detection threshold.

The detection index $\mathrm{d}$ is as follows,

$$
d=\frac{M(S+N)-M(N)}{\sigma}
$$

When $p(D)=0.5, p(F A)=10^{-4}, d \approx 4$.

The logarithm of detection index $\mathrm{d}(D T=20 \log d)$ is called Detection Threshold $D T$. The relation ${ }^{[2]}$ between Detection Threshold $(D T)$ and detection probability $(p(D))$ is as follows:

\begin{tabular}{|c|c|c|c|}
\hline$d$ & 1 & 2 & 3 \\
\hline Detection Threshold $D T$ & $0 d B$ & $6 d B$ & $9.5 d B$ \\
\hline Detection probability $p(D)$ & 0.68 & 0.954 & 0.997 \\
\hline
\end{tabular}

In sonar designing, as we often assume $D T 6 \mathrm{dBdB}$, the detection probability $p(D)$ is considered 95.4\%.In one sea test, it is a minimum probability to loss detecting the target.

The restriction of sea test route. The sonar operational range is variable with the azimuth.The criterion GJB 4258.4-2001 is the acceptance criterion for sonar operational range. It allows us three times chance to test sonar operational range. For specifically fixed target azimuth and underwater acoustic propagation environment, the sonar operational range is unique. Relatively, it is logical to test sonar operational range with invariable azimuth. The figure of sea test route is as follows, 

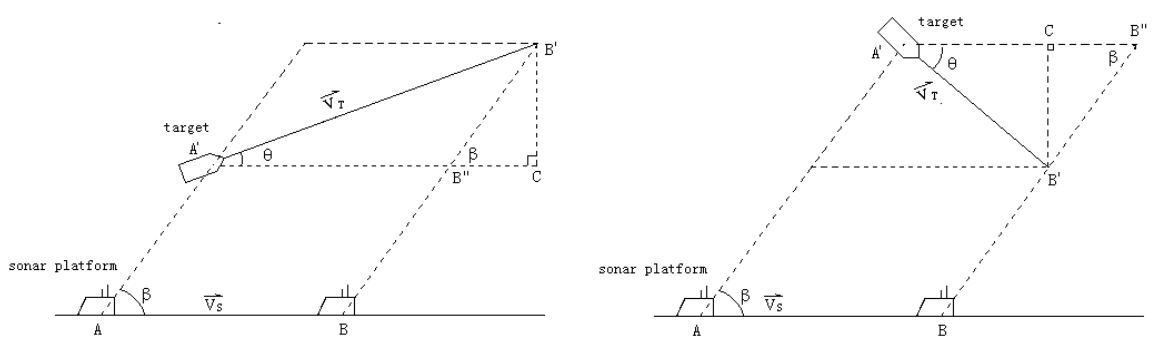

Fig.1 Sea test route to test sonar operational range with invariable azimuth

The azimuth should be restricted by the formula,

$$
V_{S}=V_{T}(\cos \theta \pm \sin \theta \cdot \operatorname{ctg} \beta)
$$

In the above formula, “+” relative to short diagonal and “-” relative to long diagonal.

Method for calculating real sonar operational range. In order to offer real sonar operational range, it is important for critical department to ensure high precision scale. We use Difference Globe Position System ( DGPS ) to position the surface ship and Underwater Position System to the submarine. The formula to calculate real sonar operational range is as follows,

$$
\begin{aligned}
& R \hat{\wedge} \sqrt{\left[x_{1}(t-\Delta t)-x_{o}(t)\right]^{2}+\left[y_{1}(t-\Delta t)-y_{o}(t)\right]^{2}} \\
& \Delta t=\frac{\sqrt{\left[x_{1}(t)-x_{0}(t)\right]^{2}+\left[y_{1}(t)-y_{0}(t)\right]^{2}}}{c}
\end{aligned}
$$

In the above formula,

$R$, real sonar operational range, $m$;

$x_{1}(\cdot) / y_{1}(\cdot), D G P S$ Gauss position data for surface ship (target), $m$;

$x_{0}(\cdot) / y_{0}(\cdot)$, Gauss position data for submarine, $m$;

$\Delta t$, modifying data for velocity of sound, $s$;

$c$, average velocity of sound, $\mathrm{m} / \mathrm{s}$.

Conversion method for sonar operational range. There are always existing differences between actual condition of sea test and limited condition of sonar operational range. In order to incarnate impartiality, it is necessary for critical department to convert the condition of sea test to limited condition of sonar operational range.

Now, we can use three models ${ }^{[3]}$ to predict Transmission Loss $T L:$ a) Parabola equation model; b)Mode wave model; c) Radial model. Parabola equation model is suit for the environment which the seabed is variable with level distance. Mode wave model is for the environment which the seabed is correspondingly fixed. Radial model is for high sound frequency ( $>400 \mathrm{~Hz}$ ). We should measure Transmission Loss $T L$ in actual sea area in order to modify the model parameters.

The conversion step for sonar operational range is as follows,

a) Measuring the parameters of target and underwater acoustic environment. These parameters are including target radiated noise level $S L_{T}$, target ambient Noise Level $S L_{W}$, Transmission Loss $T L$, ambient Noise Level $N L$ and sound speed profile.

b) Estimating the seabed acoustic parameters of underwater acoustic propagation model. These parameters are including seabed medium density $\rho$, seabed velocity of sound $c$ and absorption coefficient $\beta(f)$.

c) Calculating the sonar operational range. We utilize underwater acoustic propagation model which is modified to convert the condition of sea test to limited condition of sonar operational range. At last we can get the sonar operational range which limited by the offered condition.

\section{Limitation for tradition method to test sonar operational range}

Pass or fail is the conclusion getting from tradition method to test sonar operational range. It is joyful to developing unit. It means they have finished this sonar system developing task. On the other hand, it is too simple for sonar operator. The conclusion can't instruct sonar operator to use 
sonar system effectively. They don't know how much capability the sonar system has. In the critical report compiled by critical department, it doesn't evaluate the sonar system. Thus, it can't tell sonar operator how to use sonar system effectively in different underwater acoustic environment.

According to the mode for testing sonar operational range at the present time, the selection of target warship and sea area is optional. The chance to test don't exceed three times. If the actual sonar operational range exceeding design index of system, we don't need to convert the condition of sea test to limited condition of sonar operational range. Thus, we no longer measure the target radiated noise level, Transmission Loss $T L$, ambient Noise Level $N L$. Without these data, we can't calculate FOM in the condition of specifically target and platform.

People always think that the Transmission Loss model is not reliability and the measuring data existing error. This is why people can't believe the result of converting absolutory.

In a word, the limitation for tradition method is: a) without presenting an evaluation report for sonar system in actually detecting target; b) without instructing sonar operator to use sonar system effectively.

\section{Method to the joint of simulation and sea test based on the fixed ability of sonar system}

The FOM incarnates sonar detecting ability in the condition of specifical target and platform. Because of the changeability of underwater acoustic channel, the sonar operational range is different for a fixed FOM .

We often organize sea test in the actual underwater acoustic environment. Simulation test is a scientific predicting method for sonar operational range according to the Transmission Loss TL model and other correlative parameters. Combining the result of some typical sea test and simulation test, the ultimate goal for joint of simulation and sea test is to get the different sonar operational range in different condition.

For sonar operators of naval vessels, it is the best way to get different sonar operational range in different condition according to result of sea test absolutely. But it is not realistic. We can't arrange all kinds factors (including all different target characteristics, platform and underwater acoustic environment) to test sonar operational range. After all, the test time and outlay are limited. The method, which combines the result of some typical sea test and simulation test, is an acceptable and scientific way correspondingly.

Thinking over developing unit and sonar operators of naval vessels, the critical department should present two reports: a) one is the critical report; b) one is the evaluation report. The critical report, which expatiate the result of sea test, is pretended to developing unit. It is the basis for developing unit to manufacture the sonar system afterward. The evaluation report, which expatiate the actual ability to detect target in different condition, is pretended to naval vessels unit. It can instruct sonar operators how to use the sonar system effectively in different underwater acoustic environment.

Based on the fixed ability of sonar system, the flow of the joint of simulation and sea test is as follows: 


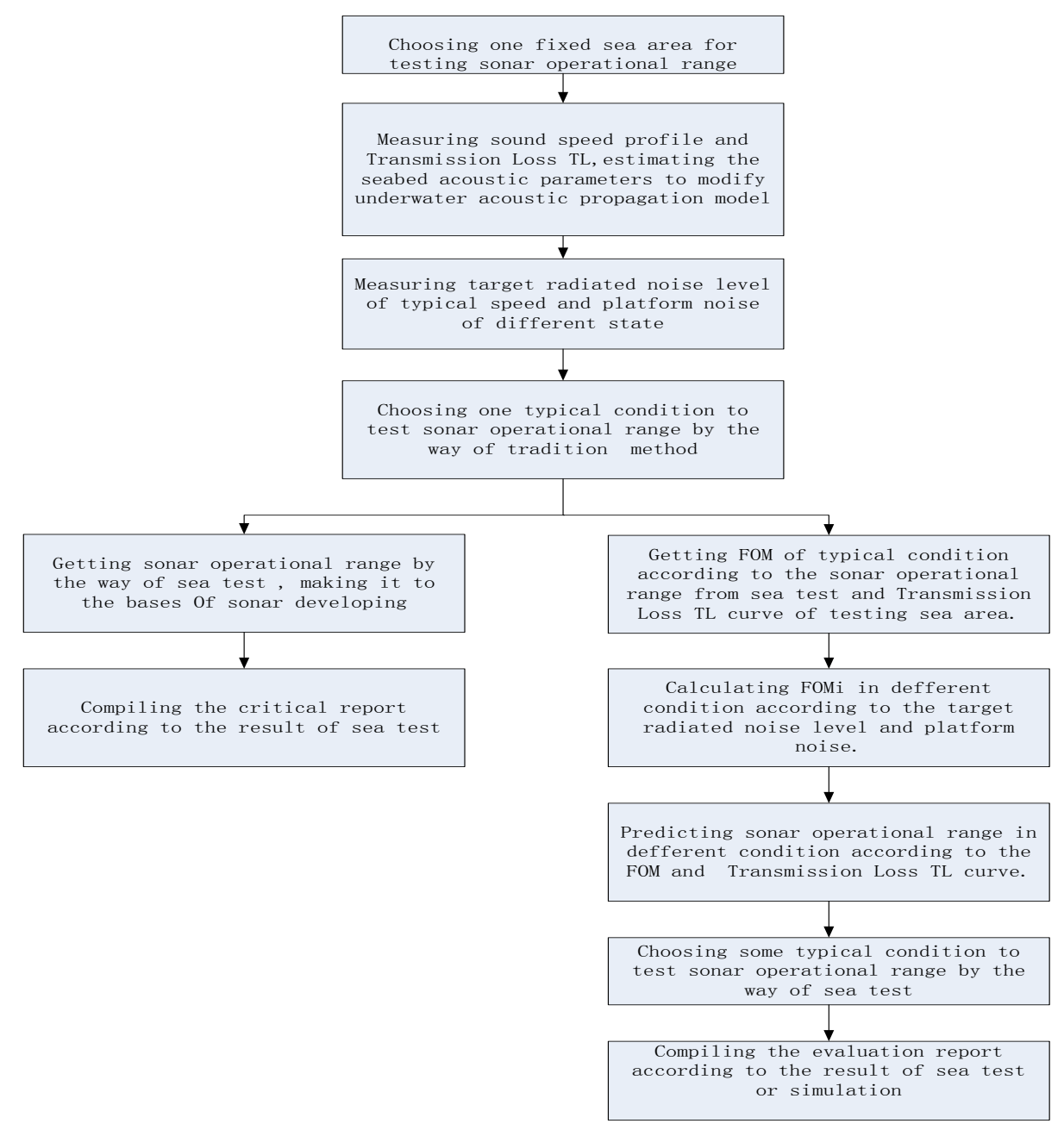

Fig.2 Flow to the joint of simulation and sea test for sonar operational range

\section{Conclusion}

This paper analyzes the factors for influencing sonar operational range and summarizes the traditional test method. Limitation for tradition method to test sonar operational range is presented too. According to the demand of sonar developing and actual using, a new test method has presented based on the fixed ability of sonar system.

\section{Rreference}

[1] URICK R J. Principles of Underwater Sound/3d edition [M], 1983, 43

[2] Liu Meng-an. Underwater Engineering[M]. ZheJiang Science and Technology Press, 2002.10

[3] Etter PC. Underwater acoustic model ---- principles, techniques and applications. London and New York: Elsevier applied science, 1991 